\title{
Approximate Expressions for the Estimation of the Four-Wave Mixing Efficiency in Slow Light Photonic Crystal Waveguides
}

\author{
Panagiotis Kanakis ${ }^{1, *}$, Thomas Kamalakis ${ }^{2}$, Thomas Sphicopoulos ${ }^{1}$, \\ ${ }^{1}$ Department of Informatics and Telecommunication, National and Kapodistrian University of Athens, \\ Panepistimioupolis, Athens GR157 84, Greece \\ ${ }^{2}$ Department of Informatics and Telematics, Harokopio University of Athens, 9 Omirou Street, \\ Athens GR176 71, Greece \\ *Corresponding author: kanakis@di.uoa.gr
}

\begin{abstract}
We present approximate analytical expressions for the estimation of the degenerate four-wave mixing conversion efficiency in slow light photonic crystal waveguides. The derived formulas incorporate the different effective modal areas and the frequency-dependent linear and nonlinear parameters of the pump, signal and idler waves. The influence of linear loss, two-photon absorption and free-carrier generation is also accounted for. Numerical solution of the coupled propagation equations is used to verify the validity of the proposed expressions under different values of the linear and nonlinear parameters of the waveguide. It is shown that the derived expressions provide an accurate estimation of the conversion efficiency and are thus expected to be very useful in the design of photonic crystal waveguides for nonlinear signal processing applications.
\end{abstract}

OCIS codes: $130.5296,190.4380$.

\section{INTRODUCTION}

Four wave mixing (FWM) is an important nonlinear phenomenon which may hold the key for many signal processing applications in future optical networks, including wavelength conversion, signal regeneration, phase inversion, optical switching and optical de-multiplexing [1-7]. Degenerate FWM occurs when part of the optical power of a signal wave can be transferred to an idler wave located at another frequency through the mediation of a strong pump wave located at a third frequency. The efficiency of the energy exchange in this process is larger when the phases of the three waves are matched, i.e. when $\Delta \varphi=2 \varphi_{p}-\varphi_{s}-\varphi_{i}$ is small, where $\varphi_{p}, \varphi_{s}, \varphi_{i}$ denote the total phase of the pump, signal and idler waves respectively. The most commonly adopted figure-of-merit which characterizes FWM is the conversion efficiency defined by,

$$
\eta \equiv \frac{P_{i}(L)}{P_{s}(0)}
$$

where $P_{s}(0)$ is the incident signal power and $P_{i}(L)$ is the idler power at the output of the waveguide of total length $L$. Nanophotonic slow-light structures such as photonic crystal waveguides (PCWs) [8] offer the possibility of achieving subwavelength light confinement, while at the same time enhancing nonlinear effects like FWM [5],[6]. Figure 1(a) shows the horizontal cross-section of a PCW formed in a triangular lattice of air holes embedded in a higher refractive index material. It was recently shown that this waveguide is particularly advantageous in FWM applications [9]. Figure 1(b) shows the dispersion relation of the waveguide along with the variation of the group index $n_{g}$ with respect to the wavelength. One observes a smooth variation of $n_{g}$ near $n_{g} \cong 60$, implying a small value of the group velocity dispersion (GVD) coefficient $\beta_{2}$, highlighted with bold lines. Usually, this area is referred to as flat-band region and in our case is defined as the wavelength range in which the group index varies $\pm 10 \%$ from the value of $n_{g}=60$.

The estimation of $\eta$ can play a crucial role in the design of the waveguide and guide us through the choice of several geometric, material and signal parameters. In the case of degenerate FWM, the evolution of the three waves is generally described by a system of coupled ordinary differential equations (ODEs) [10], the detailed form of which has recently been derived in the case of PCWs [11]. By solving this system of equations, one can in principle estimate $\eta$ using Eq. (1).

Accounting for nonlinear losses complicates the problem, rendering the derivation of an exact analytical expression extremely difficult. In semiconductor materials such as silicon, nonlinear losses usually stem from two photon absorption (TPA) and free carrier (FC) generation [3]. Self-phase modulation (SPM), cross-phase modulation (XPM) and dispersion should also be taken into account. Another complication arises from the fact that the wave parameters can exhibit substantial frequency dependence in PCWs, especially in the slow light regime. Even if the waveguide is designed to ensure a smooth linear loss and group index frequency dependence $[12,13]$, there is no guarantee that the nonlinear propagation parameters such as the effective modal areas $A$ for all three waves will be the same. In fact, recent studies argue that SPM, XPM and FWM may each perceive different values for $A$, unlike the case of a weakly guiding dielectric fiber, where such intricacies can be ignored [9].

In this work we undertake the task of providing approximate analytical expressions for the four wave mixing conversion efficiency $\eta$, when both linear and nonlinear losses affect the propagation of the three waves. The usefulness of these formulas is two-fold: First they provide significant insight into the nature of the FWM phenomenon from a theoretical point-of-view. They can also provide a target optimization function that requires much less computational time than the numerical solution of 
a)
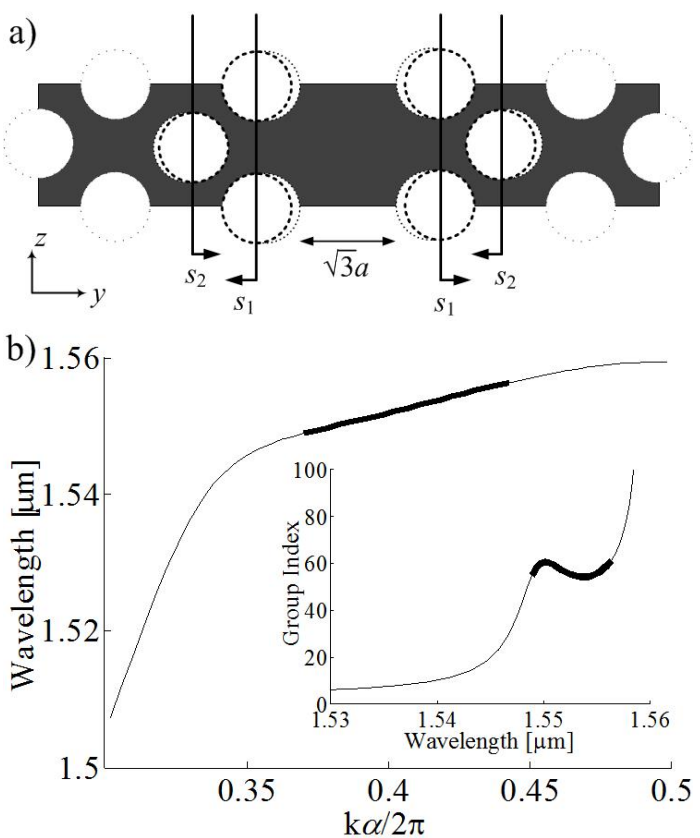

Figure 1(a): The horizontal cross section of a PCW formed in a triangular lattice of air holes embedded in a high index material (e.g. Si) with lattice constant $a_{0}=412 \mathrm{~nm}$, slab height $h=220 \mathrm{~nm}$ and hole radius $0.2913 a_{0}$. Introducing a horizontal dislocation of the holes closest to the line defect ( $1^{\text {st }}$ neighbors) by $s_{1}=0.1019 a_{0}$ and the $2^{\text {nd }}$ neighbors by $s_{2}=-0.0485 a_{0}$, results in a flat dispersion relation near $1.55 \mu \mathrm{m}$, corresponding to a group index $n_{g} \approx 60$. In Figure 1(b), the dispersion relation of the defect mode is shown. In the inset we show the variation of the mode group index with respect to the wavelength. The thick portion of the curves corresponds to the flat band region where $n_{g}$ does not change more than $\pm 10 \%$ from its specified value, $n_{\mathrm{g}}=60$.

ODEs, when designing the PCW for nonlinear signal processing applications. Unlike the design of PCWs for buffering applications [14], when optimizing the waveguide for FWM applications, one must also consider a multitude of signal parameters such as the pump-signal wavelength detuning and the incident pump power which necessitate a large number of efficiency calculations for each structure. Simple analytical expressions can therefore speed up the optimization process.

The rest of the paper is organized as follows: we first summarize the FWM model used in our calculations in section 2. In section 3, we estimate the efficiency $\eta$, initially assuming only the TPA nonlinear loss term. The derived formula incorporates the different modal effective areas as well as the frequency dependence of the nonlinear parameters. The influence of nonlinear loss attributed to FC generation is then taken into account in section 4 . To our knowledge, the derivation of $\eta$ in the presence of either or both effects has never before been sufficiently addressed in the literature. To verify the validity of our approximations we compare the values of $\eta$ obtained, against rigorous numerical solution of the coupled propagation ODEs. Good agreement is obtained for different values of wavelength detuning between the three waves as well as for various pump intensities and propagation lengths. A special treatment of the free-carrier effects is adopted in section 4.E in the case of pulsed pump. Finally, some concluding remarks are provided in section 5.

\section{DEGENERATE FWM MODEL}

Assuming that the waves are propagating in the quasi-continuous wave regime, the coupled ODEs that describe the evolution of the three waves inside the PCW are given by [11]:

$$
\begin{gathered}
\frac{d B_{p}}{d z}=-\frac{a_{p}}{2} B_{p}+T_{p}\left|B_{p}\right|^{2} B_{p}+F_{p}\left|B_{p}\right|^{4} B_{p} \\
\frac{d B_{s}}{d z}=-\frac{a_{s}}{2} B_{s}+T_{s}\left|B_{p}\right|^{2} B_{s}+F_{s}\left|B_{p}\right|^{4} B_{s}+j M_{s} B_{p}^{2} B_{i}^{*} e^{-j \Delta k z} \\
\frac{d B_{i}}{d z}=-\frac{a_{i}}{2} B_{i}+T_{i}\left|B_{p}\right|^{2} B_{i}+F_{i}\left|B_{p}\right|^{4} B_{i}+j M_{i} B_{p}^{2} B_{s}^{*} e^{-j \Delta k z}
\end{gathered}
$$

In Eqs. (2)-(4), $B_{\mu}$ and $a_{\mu}$ stand for the amplitude and the linear loss coefficients of the pump $(\mu=p)$, signal $(\mu=s)$ and idler $(\mu=i)$ waves, respectively. In Eq. (2), the coefficient $T_{p}$ encompasses the effect of SPM and TPA of the pump wave and is determined by:

$$
T_{p}=\left(j n_{2} \omega_{p} c^{-1}-\frac{1}{2} \beta_{\mathrm{TPA}}\right) S_{p}^{2} A_{p p p}^{-1}
$$

where $n_{2}$ is the nonlinear Kerr coefficient, $\omega_{p}=2 \pi c / \lambda_{p}, c$ is the speed of light in vacuum, $\beta_{\text {TPA }}$ is the TPA coefficient and $S_{p}$ stands for the slow-down factor of the pump wave, while $A_{p p p}$ is derived from [11]:

$$
A_{\rho \kappa \psi}=\frac{\left(\int_{V}\left|E_{\rho}\right|^{2} d V \int_{V}\left|E_{\rho}\right|^{2} d V \int_{V}\left|E_{\kappa}\right|^{2} d V \int_{V}\left|E_{\psi}\right|^{2} d V\right)^{1 / 2}}{a_{0} \int_{V} E_{\rho}^{*} E_{\rho}^{*} E_{\kappa} E_{\psi} d V}
$$

In Eq. (6), $V$ is the volume of the waveguide cell, $E_{\rho,} E_{\kappa,}$ and $E_{\psi}$ are the electric field components for waves $\rho, \kappa$ and $\psi$ respectively along $y$-direction and $a_{0}$ is the lattice constant of the PCW waveguide. The selection of only the transverse $y$-component of the TE guided mode of the PCW was made based on the fact that the component along the vertical $x$ direction contains only a small fraction of the incident power. In Figure 2(a), the variation of the effective modal areas pertinent to the SPM coefficient $\left(A_{p p p}\right)$, in the coupled propagation equations (2)-(4) is shown with respect to the idler wavelength for a fixed signal wavelength at $1.55 \mu \mathrm{m}$. In the same figure, we depict the values of the effective modal areas of the FWM $\left(A_{p s i}\right)$ and XPM $\left(A_{p s s}, A_{p i i}\right)$ with respect to the idlersignal detuning for a signal wavelength fixed at $1.55 \mu \mathrm{m}$. The figure indicates that, as stated in the introduction, the modal areas can exhibit strong frequency dependence even inside the flat-band region of the waveguide. It is therefore incorrect to assume the same modal area for all three waves, especially when the detuning is larger. Also the modal areas corresponding to each phenomenon may differ significantly in the case of large detuning. In Figure 2(b), the variation of the linear loss coefficient with respect to wavelengths inside the flat-band region is illustrated. The linear loss coefficients can be calculated using the model proposed in [15]-[16]. This model takes into account the geometry of the PCW and the loss coefficient in the fast-light regime $a_{f}$ which for the waveguide in question is assumed $2 \mathrm{~dB} / \mathrm{cm}$. As depicted in Figure 2(b), the linear loss coefficient is strongly wavelength dependent and hence, this should be taken into account when calculating the efficiency of FWM. 

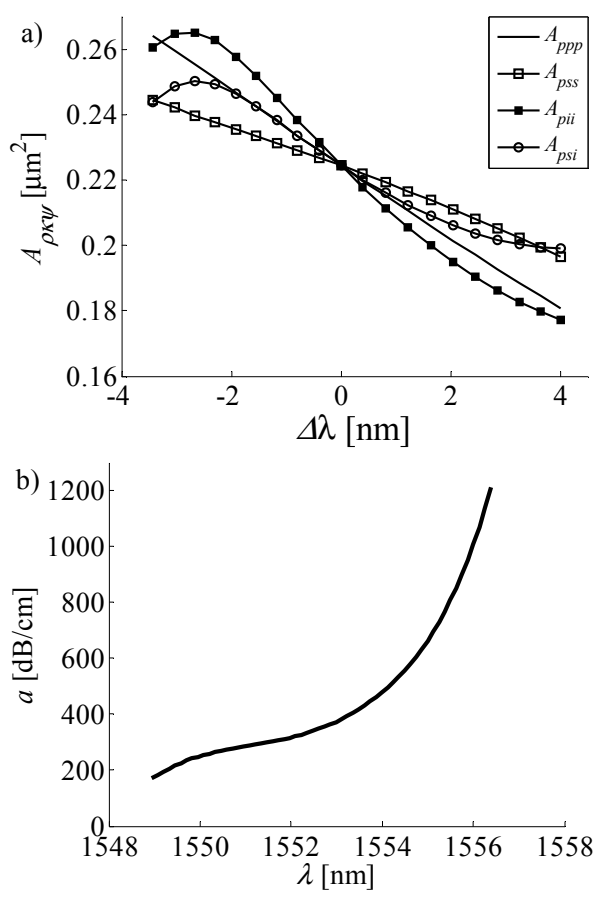

Figure 2. (a) The various effective modal areas with respect to the pumpsignal detuning. The rectangles correspond to XPM where we assume that all three modal fields in (6) are located at wavelength satisfying the expression $\lambda_{\psi}=\lambda_{k}=\lambda_{\rho}+\Delta \lambda$, and we vary $\lambda_{\rho}$. The circles correspond to FWM, i.e. $\lambda_{\psi}=\lambda_{p}+\Delta \lambda, \lambda_{k}=\lambda_{p}-\Delta \lambda$ and we again vary $\lambda_{p}$. The effective modal area for SPM is shown with plain line for fixed $\lambda_{\kappa}$ and we vary $\lambda_{\psi}$. (b) The linear loss coefficient in $\mathrm{dB} / \mathrm{cm}$ inside the flat-band region.

The coefficients $T_{s}$ and $T_{i}$ reflect the influence of XPM of the signal and idler waves with the pump as well as the effect of TPA nonlinear loss and are determined by:

$$
\begin{aligned}
& T_{s}=\left(2 j n_{2} \omega_{s} c^{-1}-\beta_{\mathrm{TPA}}\right) S_{p} S_{s} A_{p s s}^{-1} \\
& T_{i}=\left(2 j n_{2} \omega_{i} c^{-1}-\beta_{\mathrm{TPA}}\right) S_{p} S_{i} A_{p i i}^{-1}
\end{aligned}
$$

In Eqs. (7)-(8), $S_{s}$ and $S_{i}$ are the slow-down factors for the signal and idler waves respectively, $\beta_{\text {TPA }}$ is the TPA coefficient [17] while in Eqs. (2)-(4) the terms containing the coefficients $F_{\mu}$ describe the TPA-induced free carrier absorption and dispersion effects determined by [3]:

$$
F_{\mu} \cong \frac{N_{c}}{P_{p}^{2}}\left(j \frac{2 \pi}{\lambda_{\mu}} C_{1}-\frac{C_{2}}{2}\right)\left(\frac{\lambda_{\mu}}{\lambda_{0}}\right)^{2}
$$

where $P_{p}=\left|B_{p}\right|^{2}, N_{c}=\beta_{\mathrm{TPA}} S_{p}^{3} \tau_{C}\left(2 \hbar \omega_{p} A_{p p p}{ }^{2}\right) P_{p}^{2}$ is the carrier density [5,11], $\mu=p, s, i$ and for silicon waveguides, $C_{1}=-1.35 \times 10^{-27} \mathrm{~m}^{3}$, $C_{2}=1.45 \times 10^{-21} \mathrm{~m}^{2}$ and $\lambda_{0}=1550 \mathrm{~nm}$ [18]. The free carrier lifetime $\tau_{C}$, ranges from $400 \mathrm{ps}$ to $2 \mathrm{~ns}$ in Si PCWs [19]. Unless otherwise specified, we use the value of $\tau_{C}=600 \mathrm{ps}$ in our calculations. There is a third order power dependence of $N_{C}$ on the slow-down factor, as discussed in [5]. In Eqs. (3)-(4), the terms proportional to $M_{s}$ and $M_{i}$ describe the FWM interaction between the signal and the idler wave, mediated through the pump wave. These coefficients are determined by:

$$
M_{x}=n_{2} \omega_{x} c^{-1} A_{p s i}^{-1} S_{p} \sqrt{S_{s} S_{i}}
$$

In Eq. (10), $x=s, i$ for the FWM interaction of the signal and idler waves, respectively. The linear phase mismatch $\Delta k$ in Eqs. (3)-(4), is determined by $\Delta k=k_{s}+k_{l}-2 k_{p}$ where $k_{\mu}=k\left(\omega_{\mu}\right)$ is the propagation constant of the three waves and $\omega_{\mu}=2 \pi \mathrm{c} / \lambda_{\mu}, \lambda_{\mu}$ is the wavelength for wave $\mu$. A direct relation between $\Delta k$ and the dispersion characteristics can be obtained using the Taylor expansion on $\Delta k$ [20], $\Delta k \cong(\Delta \omega)^{2} \beta_{2}\left(\lambda_{\mathrm{p}}\right)+(\Delta \omega)^{4} \beta_{4}\left(\lambda_{p}\right) / 12$ where $\Delta \omega=\left|\omega_{p}-\omega_{s}\right|$, and $\beta_{2}, \beta_{4}$ are the dispersion coefficients of second and fourth order, respectively. It is therefore clear that dispersive designs will result in larger $\Delta k$, further downgrading the FWM conversion efficiency.

There are two assumptions implied in Eqs. (2)-(4) that should be stated. First of all, we assume that the pump power $P_{p}=\left|B_{p}\right|^{2}$ is significantly larger than the power of the idler and the signal waves $P_{i}=\left|B_{i}\right|^{2}$ and $P_{s}=\left|B_{s}\right|^{2}$, respectively. In actual experiments, the pump power is usually more than ten times higher than the signal power, hence XPM and FWM effects in Eq. (2) can be ignored compared to SPM of the pump-wave. This also suggests that SPM effects in Eqs. (3)-(4) can be neglected in the presence of the much stronger XPM interaction between the pump and the signal or idler waves. The incident signal power is assumed, $P_{s}(0)=0.5 \mathrm{~mW}$ throughout our calculations. Another assumption commonly adopted in FWM efficiency calculations is that the waves are propagating in a quasi-CW regime and hence dispersion effects can be ignored. This can be justified by comparing the propagation distances with the dispersion length $L_{D}=T_{0}^{2} / \beta_{2} \mid$ (where $T_{0}$ is the root mean square pulse width). For the PCW of Figure 1(a) we obtain $\left|\beta_{2}\right| \leq 24.7 \mathrm{psec}^{2} / \mathrm{mm}$ inside the flatband region (thick portion of Figure 1(b)). For a signal of $R_{b}=10 \mathrm{~Gb} / \mathrm{s}$, one can choose the full width half maximum pulse width equal to $1 /\left(4 R_{b}\right)=25 \mathrm{ps}$ [14] which for Gaussian pulses corresponds to $T_{0} \cong 15 \mathrm{ps}$ and a dispersion length equal to $L_{D} \cong 9 \mathrm{~mm}$. The dispersion length is therefore much larger than the propagation distances considered here $(L \leq 500 \mu \mathrm{m})$ and as a consequence, the dispersion terms in the propagation equations are neglected for the three waves. Of course dispersion effects are still accounted for in the phase mismatch $\Delta k$. Using a similar reasoning, we deduce that if a pulsed pump is used, dispersion effects can be ignored in the pump propagation equation as long as the pulse width is $T_{0}>3.5 \mathrm{ps}$.

Before turning our attention to nonlinear loss, it is interesting to state the approximate expression for the FWM efficiency $\eta$ in the presence of linear loss only:

$$
\eta=\frac{\omega_{i}}{\omega_{s}}\left(1+\frac{\kappa^{2}}{4 g^{2}}\right) \sinh ^{2}(g L) e^{-a_{i} L}
$$

This equation is based on the expression used previously in the literature [20-22] but we have now taken into account the variation of the various parameters with respect to wavelength. In Eq. (11), $L$ is the waveguide length, and $\kappa$ and $g$ are the total phase mismatch and parametric gain determined by,

$$
\kappa=\Delta k+\frac{2 n_{2}}{c} \bar{P}_{p}\left(\frac{\omega_{s}}{A_{p s s}}+\frac{\omega_{i}}{A_{p i i}}-\frac{\omega_{p}}{A_{p p p}}\right)
$$




$$
g=\sqrt{\left(\frac{n_{2} S_{p}}{c A_{p s i}}\right)^{2} S_{i} S_{p} \omega_{i} \omega_{s} \bar{P}_{p}^{2}-\kappa^{2} / 4}
$$

In Eqs. (12)-(13), $\bar{P}_{p}$ is the average pump power across the waveguide given by:

$$
\bar{P}_{p} \equiv \frac{1}{L} \int_{0}^{L} P_{p}(z) d z=P_{p}(0) \frac{1-e^{-a L}}{a L}
$$

The expression of the FWM conversion efficiency in Eq. (11) can be used in the case where the TPA nonlinear losses can be suppressed. This is usually accomplished concerning materials possessing lower TPA coefficient $\left(\beta_{\text {TPA }}\right)$ frequently referred to as TPA-free materials [23]-[25], which are currently receiving increased attention in signal processing applications. Although this result is known and frequently used in the literature, its derivation provides the underlying idea used in the next sections. To derive Eq. (11), one uses the lossless formula for FWM efficiency [10] and a) accounts for the losses experienced by the idler wave multiplying by the factor $\exp \left(-a_{i} L\right)$ in Eq. (11) and b) neglects the variation of the pump power assuming that the pump power is equal to the average pump power $\bar{P}_{p}$. In the case where the pump wave experiences nonlinear losses, the calculation of $\bar{P}_{p}$ is not straightforward. One must also account for the nonlinear loss experienced by the idle wave which is mainly due to the existence of the pump wave.

In this work we focus on the estimation of the FWM conversion efficiency in the presence of both linear and nonlinear losses. It is worth mentioning that in the linear case, the exact FWM conversion efficiency formula could be derived in terms of Whittaker confluent hypergeometric functions [26]. Although this is a very useful result, one should note that the estimation of Whittaker functions is usually quite involved and the corresponding computational cost can be comparable to that of solving the ODEs in the first place.

\section{INCLUSION OF TPA-INDUCED LOSS.}

In this section we discuss the analytic estimation of the FWM efficiency in PCWs when both the linear and the nonlinear TPA loss are included in Eqs. (2)-(4). TPA is typically accompanied by free-carrier generation causing an additional absorption and dispersion term expressed by the real and imaginary parts of $F_{p, s, i}$ respectively in Eqs. (2)-(4). As discussed by several authors, FC effects can be significantly reduced when either a low repetition/ low duration pulsed pump is used [21], or when an external DC field is applied driving the free carriers away from the center of the waveguide [27], [28]. Under this assumption, the evolution of the three waves is governed by setting $F_{p, s,}=0$ in Eqs. (2)-(4).

To derive an expression for the efficiency, we start by estimating the power of the pump wave, $P_{p}(z)=\left|B_{p}(z)\right|^{2}$. Substituting $B_{p}(z)=P_{p}(z)^{1 / 2} \exp \left(j \varphi_{p}\right)$ in Eq. (2) and using the fact that $F_{p}=0$, it is straightforward to show that:

$$
\frac{d P_{p}}{d z}=-\left[a_{p}+2 \operatorname{Re}\left\{T_{p}\right\} P_{p}\right] P_{p}
$$

The above equation can be readily solved to yield:
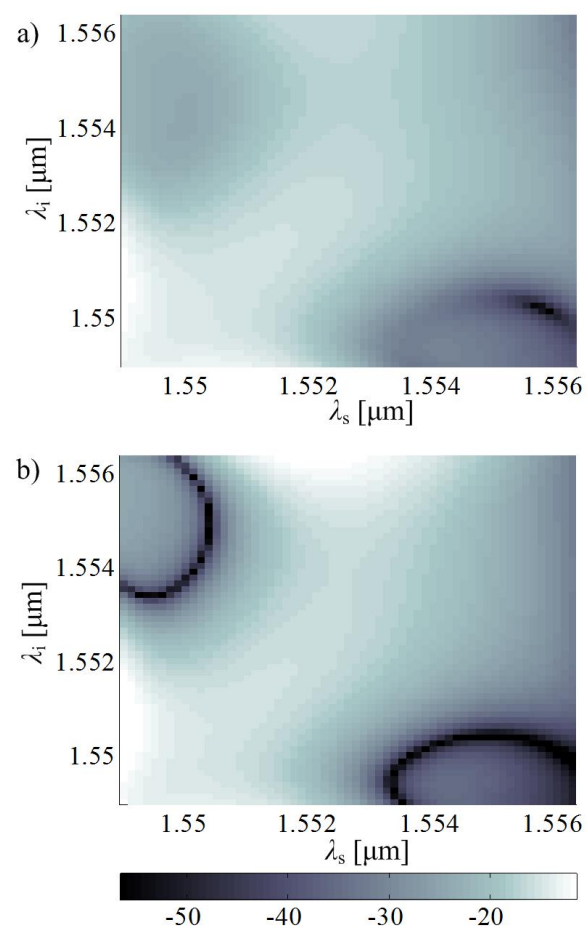

Figure 3. Wavelength dependence of FWM conversion efficiency (a) numerically calculated and (b) estimated using (22), assuming $P_{p}(0)=2 \mathrm{~W}$ and $L=200 \mu \mathrm{m}$.

$$
P_{p}(z)=\frac{P_{p}(0) e^{-a_{p} z}}{1+P_{p}(0) \beta_{\mathrm{TPA}} S_{p}^{2}\left(1-e^{-a_{p} z}\right) /\left(a_{p} A_{p p p}\right)}
$$

where we have substituted the value of $\operatorname{Re}\left\{T_{p}\right\}$ determined by Eq. (5). The average pump power $\bar{P}_{p}$ is calculated by substituting Eq. (16) in Eq. (14), yielding:

$$
\bar{P}_{p}=\frac{A_{p p p}}{\beta_{\mathrm{TPA}} S_{p}^{2} L} \ln \left(1+\frac{\beta_{\mathrm{TPA}} S_{p}^{2} P_{p}(0)}{a_{p} A_{p p p}}\left[1-e^{-a_{p} L}\right]\right)
$$

As stated in the previous section, we can assume that the propagation of the signal and idler waves is lossless and that the pump power is constant and equal to $\bar{P}_{p}$, i.e.:

$$
\begin{aligned}
& \frac{d B_{s}}{d z}=j \operatorname{Im}\left\{T_{s}\right\}\left|B_{p}\right|^{2} B_{s}+j M_{s} \bar{P}_{p} B_{i}^{*} e^{j \Delta k z} \\
& \frac{d B_{i}}{d z}=j \operatorname{Im}\left\{T_{i}\right\}\left|B_{p}\right|^{2} B_{i}+j M_{i} \bar{P}_{p} B_{s}^{*} e^{j \Delta k z}
\end{aligned}
$$

This lossless version of the coupled propagation equations can be solved exactly by generalizing the method used in [10] and properly taking into account the different coefficients for each wave. The lossless FWM efficiency $\eta_{0}$ obtained turns out to be:

$$
\eta_{0}=\frac{\omega_{i}}{\omega_{s}}\left(1+\frac{\kappa^{2}}{4 g^{2}}\right) \sinh ^{2}(g L)
$$



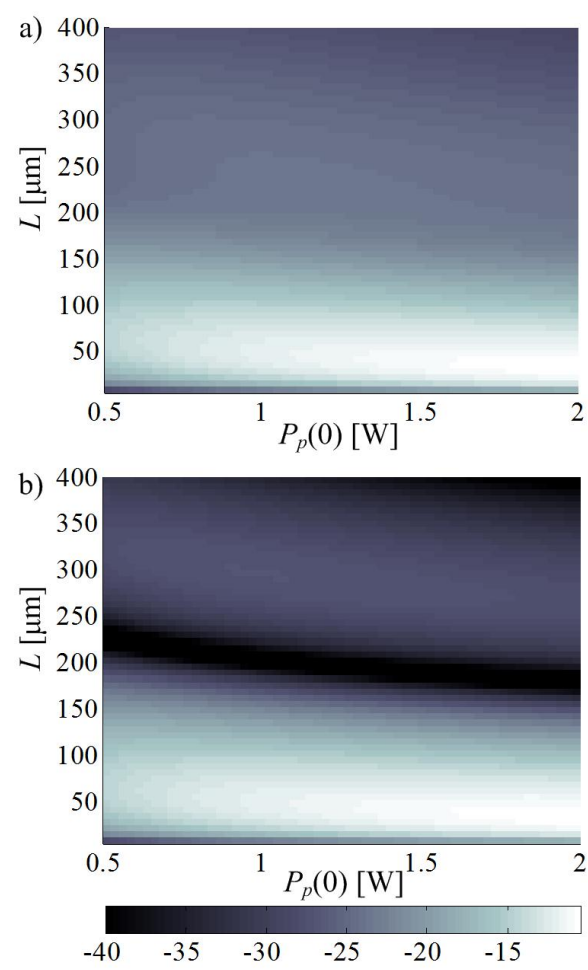

Figure 4. FWM conversion efficiency with respect to the waveguide length and the incident pump power calculated (a) numerically, and (b) analytically using (22). The wavelength of the signal and the idler waves are considered fixed at $\lambda_{\mathrm{s}}=1553.6 \mathrm{~nm}$ and $\lambda_{\mathrm{i}}=1549.3 \mathrm{~nm}$, respectively.

It is worth noticing that, in Eq. (20) the total phase mismatch $\kappa$ and the parametric gain $g$ are still given by Eqs. (12) and (13), respectively since the TPA term is a purely loss term but this time $\bar{P}_{p}$ is given by Eq. (17) instead of Eq. (14). As outlined in

Section 2, we next need to account for the power loss experienced by the idler wave as it propagates through the waveguide (in the absence of FWM). Using Eq. (4) and the fact that $P_{i}(z)=\left|B_{i}(z)\right|^{2}$, we can show that:

$$
\frac{d P_{i}}{d z}=-\left(a_{i}+2 \operatorname{Re}\left\{T_{i}\right\} P_{p}\right) P_{i}
$$

Dividing by $P_{i}$ and integrating with respect to $z$, we can show that the losses of the idler wave, $P_{i}(L) / P_{i}(0)$, are equal to $\exp \left(-a_{i} L-2 \operatorname{Re}\left\{T_{i}\right\} \bar{P}_{p} L\right)$. Therefore, we can derive an approximate formula for the FWM efficiency in the lossy case by accounting for the idler wave loss, in the following expression,

$$
\eta=\left(\frac{\omega_{i}}{\omega_{s}}\right)\left(1+\frac{\kappa^{2}}{4 g^{2}}\right) \sinh ^{2}(g L) e^{-a_{i} L-2 \operatorname{Re}\left\{T_{i}\right\} \bar{P}_{p} L}
$$

We compare the values obtained by Eq. (22) against the numerical solution of the propagation equations Eqs. (2)-(4) using an embedded Runge-Kutta scheme [29] for the waveguide in Figure 1. In our calculations, the incident pump power is $P_{p}(0)=2 \mathrm{~W}$ and the waveguide length is set at $L=200 \mu \mathrm{m}$. We also assume that the TPA coefficient $\beta_{\text {TPA }}$ is $2 \times 10^{-11} \mathrm{~m} / \mathrm{W}$ comparable to that of $\mathrm{Al}_{x} \mathrm{Ga}_{1-x} \mathrm{As}$ [30]. This TPA coefficient is somewhat higher than that of silicon $\left(\beta_{\text {TPA }} \cong 5 \times 10^{-12} \mathrm{~m} / \mathrm{W}\right)$ and is assumed in order to increase the TPA loss level compared to the linear loss level (for
$B_{\text {TPA }}=5 \times 10^{-12} \mathrm{~m} / \mathrm{W}$, the average TPA loss, concerning all possible signal-idler wavelength detunings inside the flat-band wavelength range, is only $-2 \mathrm{~dB}$, but increasing $b_{\text {TPA }}$ to $2 \times 10^{-11} \mathrm{~m} / \mathrm{W}$ results in an average TPA loss of almost $-6.5 \mathrm{~dB}$, for an incident pump power of $P_{p}(0)=2 \mathrm{~W}$. The average linear loss level for the same wavelength range is almost $-8 \mathrm{~dB}$ ).

In Figure 3, we compare the values obtained by Eq. (22) against numerical solution of the ODEs with respect to the wavelength of the signal and the idler waves. We visually infer that overall the approximate formula provides an adequate description for medium-to-high efficiency values which are important from a practical point of view. There is some discrepancy in low value regions (i.e. where the ODEs efficiency is $\leq-10 \mathrm{~dB}$ than the maximum efficiency value obtained by the ODEs, $\eta_{\max }=-11.4 \mathrm{~dB}$ ). To quantify the error in the approximation, we calculated the average error $e_{5}$ and $e_{10}$ between the numerical and the analytical efficiency (measured in $\mathrm{dB}$ ) for wavelength combinations in which the ODEs efficiency is not lower than $-5 \mathrm{~dB}$ and $-10 \mathrm{~dB}$ compared to $\eta_{\max }$, respectively. In the case of Figure 3, we obtain $e_{5}=0.35 \mathrm{~dB}$ and $e_{10}=1.1 \mathrm{~dB}$ implying very good agreement for efficiency values of practical interest. In Figure 4(a) and (b), we compare the FWM efficiency with respect to the waveguide length and the incident pump power obtained by the ODEs and the analytical approximation in Eq. (22). In these calculations, we have assumed that the signal and idler waves are located at fixed wavelengths, $\lambda_{\mathrm{s}}=1553.6 \mathrm{~nm}$ and $\lambda_{\mathrm{i}}=1549.3 \mathrm{~nm}$ respectively resulting a pump-signal detuning of $\Delta \lambda \cong 2 \mathrm{~nm}$. Inspecting Figure 4 , we again deduce that at medium-to-high efficiency values, the analytical approximation agrees well with the numerical result. However as the efficiency becomes smaller, the analytical formula predicts smaller efficiencies than the ODEs. To explain this discrepancy observed in Figure 3 and Figure 4 at small efficiency values, we examine Eq. (22), according to which, the analytical approximation for the efficiency can become very small for $g L \cong j \pi m$ where $m$ is an integer, due to the $\sinh (g L)$ factor. Taking into account Eq. (13) we deduce that if $\bar{P}_{p}$ is chosen large enough that the first term under the root is smaller than $\kappa^{2} / 4$ then $g$ will be imaginary. Hence as $L$ increases, there will be an infinite number of values of $L=L_{m}$ for which $g L_{m}$ will be multiple of $j \pi$ and hence the approximate $\eta$ will be zero. For these values of $L$, the total linear and non-linear phase mismatch is such that the waves interfere destructively at the end of the waveguide. In the actual case where the pump power varies with distance as a result of the linear and nonlinear loss, the phase relation will turn out more complicated and in general one can no longer expect to find values of $L$ for which the efficiency is exactly zero. This is why there are no black regions in Figure 4(a) in which $\eta$ is very close to zero. We do however point out that in these regions, the actual ODEs efficiency is much smaller that its maximum value anyway, so this error in the approximation has little bearing from a practical point-of-view.

\section{INCLUSION OF FREE-CARRIER EFFECTS.}

If no measures are taken, the free-carrier generation can severely limit the FWM conversion efficiency. When this effect is included, then deriving an analytical approximation for $\eta$ is much more involved. For one thing, the pump power cannot be obtained in exact form as in the previous cases. To see this, we again use the transformation $B_{p}(z)=P_{p}(z)^{1 / 2} \exp \left(j \varphi_{p}\right)$ in Eq. (2) but this time the term proportional to $\left|B_{p}\right|{ }^{4} B_{p}$ corresponding to the free carrier 
generation is retained. We obtain the following equation for the pump power evolution:

$$
\frac{d P_{p}}{d z}=-\left(a_{p}+2 \operatorname{Re}\left\{T_{p}\right\} P_{p}+2 \operatorname{Re}\left\{F_{p}\right\} P_{p}^{2}\right) P_{p}
$$

The differential equation in Eq. (23) is simply not amenable to analytical solution but a number of alternatives can be pursued. In the following sub-sections, we present two alternative methods for obtaining $P_{p}(z)$ which can be used in the estimation of $\eta$.

\section{A. Loss independence approximation}

First we may assume that the three loss types (free-carrier absorption, TPA and linear loss) act independently and that the overall pump loss can be approximated by the product of the three loss factors. The loss factor for the linear loss is simply the exponential $\exp \left(-a_{p} z\right)$, while for the TPA-induced factor, it can be obtained by Eq. (21) in the limit $a_{p} \rightarrow 0$ and is equal to $\left[1+P_{p}(0) \beta_{\mathrm{TPA}} S_{p}^{2} \mathrm{z} / A_{\mathrm{ppp}}\right]^{-1}$. In the case of free-carrier generation, we simply solve Eq. (23) assuming that $a_{p}=0$ and $T_{p}=0$, i.e. $\mathrm{d} P_{p} / \mathrm{d} z=-2 \operatorname{Re}\left\{F_{p}\right\} P_{p}^{3}$ which yields $P_{p}(z) / P_{p}(0)=\left[1+4 P_{p}(0)^{2} \operatorname{Re}\left\{F_{p}\right\} z\right]^{-1 / 2}$. Hence, the pump power can be approximated by:

$$
P_{p}(z)=P_{p}(0) \frac{e^{-a_{p} z}}{\left(1+K_{1} z\right)\left(1+K_{2} z\right)^{1 / 2}}
$$

where we have defined the parameters $K_{1}=P_{p}(0) \beta_{\mathrm{TPA}} S_{p}{ }^{2} /\left(A_{p p p}\right)$ and $K_{2}=4 P_{p}(0)^{2} \operatorname{Re}\left\{F_{p}\right\}$. We now use this expression to obtain the average pump power. To facilitate the calculations, the linear loss coefficient is approximated by a first order polynomial, i.e. $\exp \left(-a_{p} z\right) \cong e_{0}+e_{1} z$. The coefficients $e_{0}$ and $e_{1}$ can be obtained so that the difference between the exponential and its first order approximation is minimum in the least-square sense inside $[0, L]$, in which case we find that:

$$
\begin{gathered}
e_{0}=2 l_{0}^{-1}\left[\left(e^{-l_{0}}+2\right)+3 l_{0}^{-1}\left(e^{-l_{0}}-1\right)\right] \\
e_{1}=-6 l_{0}^{-1} L^{-1}\left[\left(e^{-l_{0}}+1\right)+2 l_{0}^{-1}\left(e^{-l_{0}}-1\right)\right]
\end{gathered}
$$

with $l_{0}=a_{p} L$. Adopting this first order approximation for the exponential, we may readily obtain a closed form formula for the average pump power,

$$
\bar{P}_{p}=\left[\frac{2\left(e_{1}-e_{0} K_{1}\right)}{L K_{1}^{3 / 2} \sqrt{K_{1}-K_{2}}} \tanh ^{-1}\left(\frac{\sqrt{K_{1}} \sqrt{1+K_{2} z}}{\sqrt{K_{1}-K_{2}}}\right)+\frac{2 e_{1} \sqrt{1+K_{2} z}}{L K_{1} K_{2}}\right]_{0}^{L}
$$

where $[f(z)]_{b}^{d}=f(d)-f(b)$. We may also obtain an expression for the average square pump power which will be useful in our subsequent analysis,

$$
\begin{gathered}
\overline{P_{p}^{2}}=\frac{1}{L}\left[-\frac{\left(e_{1}-e_{0} K_{1}\right)^{2}}{K_{1}^{2}\left(K_{1} z+1\right)\left(K_{1}-K_{2}\right)}+\right. \\
\left.+\left\{\frac{\left(e_{0} K_{1}-e_{1}\right)\left[e_{1}\left(2 K_{1}-K_{2}\right)-e_{0} K_{1} K_{2}\right]}{K_{1}^{2}}+\frac{\left(e_{1}-e_{0} K_{2}\right)^{2}}{K_{2}}\right\} \frac{\ln \left(1+K_{1} z\right)}{\left(K_{1}-K_{2}\right)^{2}}\right]_{0}^{L}
\end{gathered}
$$

\section{B. Ignoring the TPA losses}

An alternative is to solve Eq. (23) in the case where the nonlinear loss is dominated by the free-carrier absorption, i.e. $\operatorname{Re}\left\{F_{p}\right\} P_{p} \gg>\operatorname{Re}\left\{T_{p}\right\}$, in which case,

$$
\frac{d P_{p}}{d z} \cong-\left(a_{p}+2 \operatorname{Re}\left\{F_{p}\right\} P_{p}^{2}\right) P_{p}
$$

This equation can be solved analytically to yield:

$$
P_{p}(z)=\frac{P_{p}(0) e^{-a_{p} z}}{\left(1+\delta\left(1-e^{-2 a_{p} z}\right)\right)^{1 / 2}}
$$

where $\delta$ is given by,

$$
\delta=2 a_{p}^{-1} \operatorname{Re}\left\{F_{p}\right\} P_{p}(0)^{2}
$$

The expression in Eq. (30) has also been derived elsewhere [28],[31-32]. Integrating with respect to $z$ and dividing with the waveguide length, we readily obtain the following expressions for the average pump power and the average square pump power:

$$
\begin{gathered}
\bar{P}_{p}=-\frac{P_{p}(0)}{a_{p} L \sqrt{\delta}}\left\{\sin ^{-1}\left(\frac{e^{-a_{p} L}}{\sqrt{\delta^{-1}+1}}\right)-\sin ^{-1}\left(\frac{1}{\sqrt{\delta^{-1}+1}}\right)\right\} \\
\overline{P_{p}^{2}}=\frac{P_{p}(0)^{2}}{2 a_{p} \delta} \ln \left(1+\delta\left(1-e^{-2 a_{p} L}\right)\right)
\end{gathered}
$$

\section{Efficiency calculations}

Once the pump power is obtained by either one of the two methods discussed above, we can proceed to the estimation of the approximate efficiency in a similar manner to the previous sections. The lossless problem is described by equations (3)-(4) retaining only the imaginary parts of the terms corresponding to XPM, FWM and FC generation. We arrive at the same expression for $\eta_{0}$ as before in Eq. (20), except that the total phase mismatch $\kappa$ is replaced by $\kappa_{\text {tot }}$ which is determined by:

$$
\kappa_{\text {tot }}=\kappa+\operatorname{Im}\left\{F_{s}+F_{i}-2 F_{p}\right\} \overline{P_{p}^{2}}
$$

where $\kappa$ is determined by Eq. (12) substituting the expression for $\bar{P}_{p}$ derived in either sections 4 .A or 4.B. The second term in the sum is the surplus mismatch due to the free carrier dispersion. We also proceed to calculate the losses experienced by the idler wave, in the absence of FWM. The idler power is described by:

$$
\frac{d P_{i}}{d z}=-\left(a_{i}+2 \operatorname{Re}\left\{T_{i}\right\} P_{p}+2 \operatorname{Re}\left\{F_{i}\right\} P_{p}^{2}\right) P_{i}
$$

Solving (35), we can estimate the loss $l_{i}$ experienced by the idler wave,

$$
l_{i}=\frac{P_{i}(L)}{P_{i}(0)}=\exp \left(-a_{i} L+2 L \operatorname{Re}\left\{T_{p}\right\} \bar{P}_{p}+2 L \operatorname{Re}\left\{F_{i}\right\} \overline{P_{p}^{2}}\right)
$$



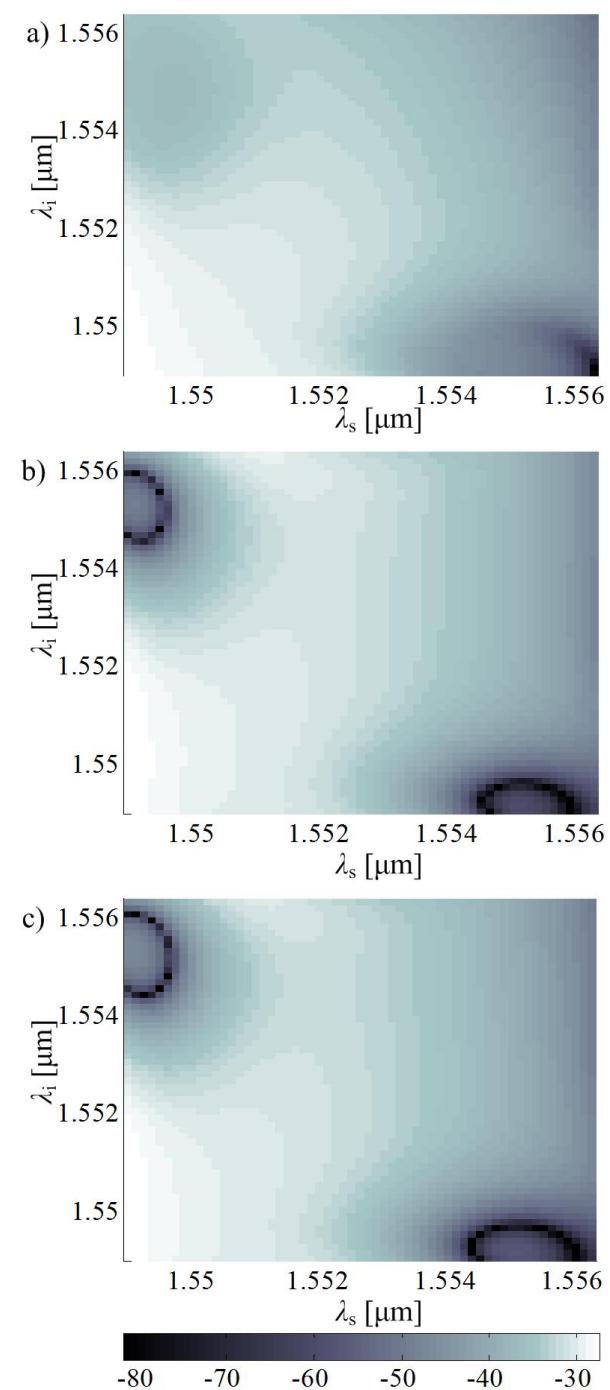

Figure 5. Wavelength dependence of FWM conversion efficiency when the FC effects are included, (a) numerically calculated, (b) by treating the three loss mechanisms independently and (c) by ignoring the TPA loss term. The waveguide length is $L=200 \mu \mathrm{m}$ and the incident pump power $P_{p}(0)=2 \mathrm{~W}$.

The above equation dictates that the losses of the idler wave can be estimated once the average power $\bar{P}_{p}$ and the average square power $\overline{P_{p}^{2}}$ are calculated. Multiplying the lossless efficiency with the idler losses in Eq. (36), we obtain the approximate expression for the FWM conversion efficiency,

$$
\eta=l_{i}\left(\frac{\omega_{i}}{\omega_{s}}\right)\left(1+\frac{\kappa_{\mathrm{tot}}^{2}}{4 g^{2}}\right) \sinh ^{2}(g L)
$$

where $g$ is the parametric gain obtained by Eq. (13) only this time the total phase mismatch will be given by Eq. (34) instead of Eq. (12).

\section{Validation of the analytical solution}

In this sub-section we validate the results obtained by the analytical formula (37) considering both alternative methods for estimating the pump power. In Figure 5, we compare the FWM conversion efficiency obtained by Eq. (37) against numerical solution with respect to the wavelength of the signal and the idler waves, assuming $B_{\mathrm{TPA}}=5 \times 10^{-12} \mathrm{~m} / \mathrm{W}, \tau_{\mathrm{C}}=600 \mathrm{ps}, \quad L=200 \mu \mathrm{m}$ and $P_{p}(0)=2 \mathrm{~W}$. Figure 5(b) and (c) show the approximate FWM conversion efficiency obtained when the pump power estimated by the methods considered in Section 4.A and 4.B, respectively. As shown in Figure 5, an overall good agreement is obtained between the numerical and the analytical solution for medium to high efficiency values. The average error for $\left(\lambda_{i}, \lambda_{s}\right)$ combinations for which the ODE efficiency is not smaller than $-5 \mathrm{~dB}$ and $-10 \mathrm{~dB}$ than $\eta_{\max }=-21 \mathrm{~dB}$ is $e_{5}=0.53 \mathrm{~dB}$ and $e_{10}=2.03 \mathrm{~dB}$ in the case of Figure 5(b). In Figure 5(c) where the TPA loss is neglected, we obtain slightly better results, $e_{5}=0.28 \mathrm{~dB}$, and $e_{10}=1.95 \mathrm{~dB}$. We have confirmed that for these combinations of $\left(\lambda_{i}, \lambda_{s}\right)$ the value of the TPA loss is much lower than the FC-induced loss and it is therefore not surprising that the method of Section 4.B yields better results. In Figure 6, we compare the FWM conversion efficiency to the numerical solution, for various waveguide
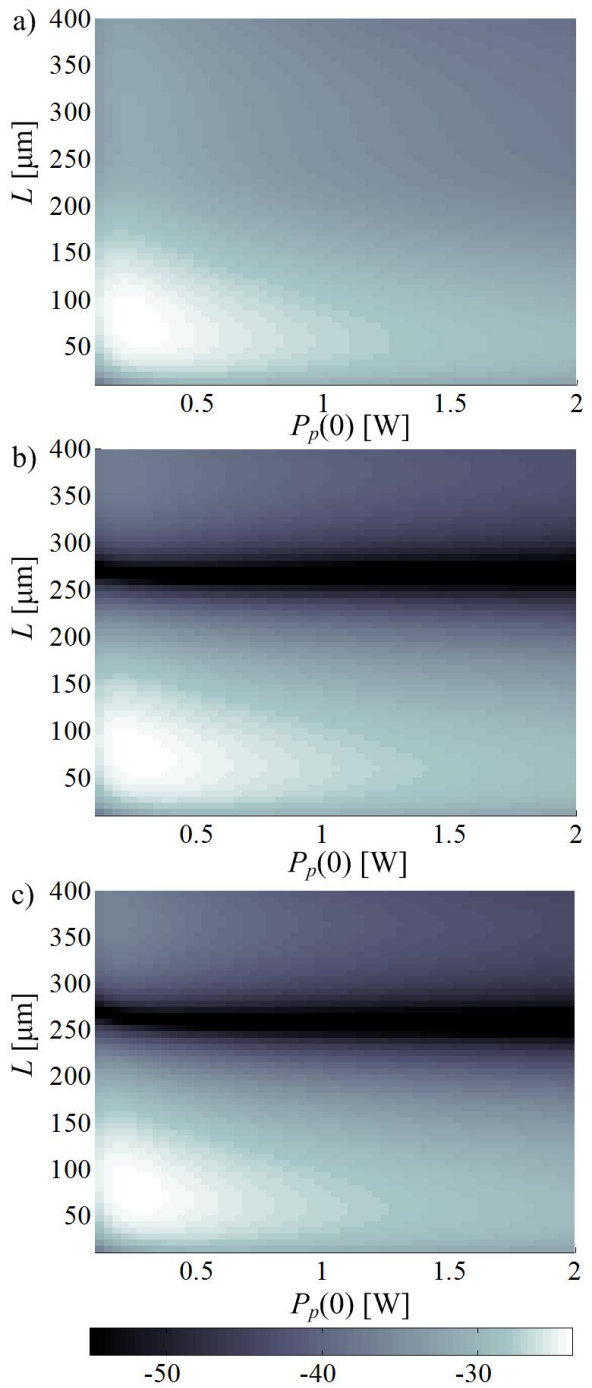

Figure 6. FWM conversion efficiency with respect to the waveguide length, $L$ and the incident pump power, $P_{p}(0)$ incorporating the FC effects is calculated (a) numerically, (b) by treating the three loss mechanisms independently and (c) by ignoring the TPA loss term. The wavelength of the signal and the idler waves are considered fixed at $\lambda_{\mathrm{s}}=1553.6 \mathrm{~nm}$ and $\lambda_{1}=1549.3 \mathrm{~nm}$, respectively. 

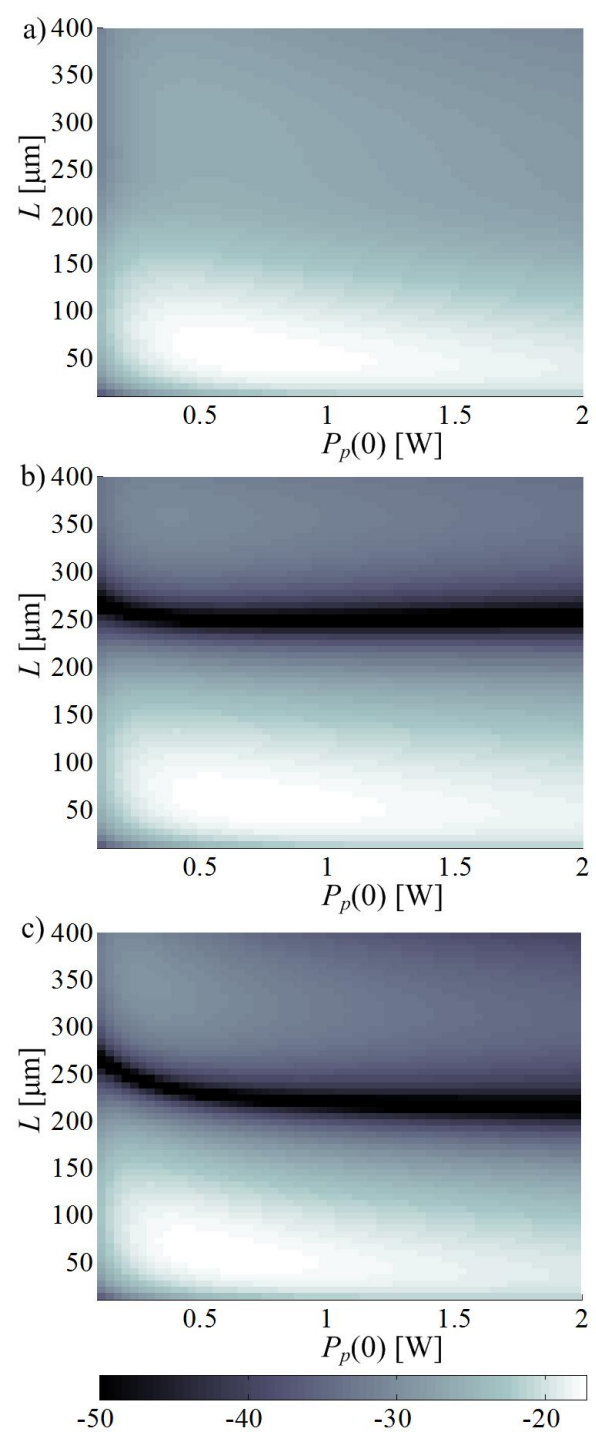

Figure 7. FWM conversion efficiency assuming that the three loss mechanisms have comparable impact to the propagating light with respect to the waveguide length, $L$ and the incident pump power, $P_{p}(0)$ calculated (a) numerically, (b) by treating the three loss mechanisms independently and (c) by ignoring the TPA loss term.

lengths and incident pump powers, when the pump power is estimated by the methods outlined in sections 4.A and 4.B, respectively. We assume that the wavelength detuning between the pump and signal waves is fixed at $\Delta \bumpeq \cong 2 \mathrm{~nm}$ (for $\lambda_{\mathrm{s}}=1553.6 \mathrm{~nm}$ and $\lambda_{i}=1549.3 \mathrm{~nm}$ ). We obtain $e_{5}=0.27 \mathrm{~dB}$ and $e_{10}=3.27 \mathrm{~dB}$ in the case of Figure $6(\mathrm{~b})$ and $e_{5}=0.21 \mathrm{~dB}$ and $e_{10}=3.26 \mathrm{~dB}$ in the case of Figure 6(c). We note that the analytical formulas provide excellent results near $\eta_{\max }$, but there is a larger error for smaller efficiency values. This is a consequence of the fact that the variations of $\eta$ are now smoother. For example, as shown in Figure 5(a), the regions of $\left(\lambda_{i}, \lambda_{s}\right)$ which are considered in the estimation of $e_{10}$ (i.e. where the efficiency is no less than $-10 \mathrm{~dB}$ lower than its maximum value) cover most of the surface plot and correspond to rather large wavelength detuning, $\Delta$.

The results obtained in Figures 5 and 6 are calculated based on state-of-the-art fast-light linear loss levels and values of $\beta_{\text {TPA }}$ corresponding to silicon. If no measures are taken to limit freecarrier effects, then these dominate the nonlinear losses. We would however like to validate our theory in a case where all three loss factors (linear, TPA and free-carrier induced) are comparable. To this end we recalculate the three efficiencies for the same linear loss level assuming $\beta_{\mathrm{TPA}}=2 \times 10^{-11} \mathrm{~m} / \mathrm{W}$ and $\tau_{C}=15 \mathrm{ps}$ [28]. Assuming a range of values [0.1W $2 \mathrm{~W}]$ for the initial pump power $P_{0}=P_{p}(0)$ and $[5 \mu \mathrm{m} 400 \mu \mathrm{m}]$ for the waveguide length $L$, we have estimated the loss for each of the three contributions and we found that each one has an average value of about $-6 \mathrm{~dB}$ in the absence of the other two loss factors. From a practical point-ofview, the choice of these parameters corresponds to a material with higher TPA nonlinearity and taking measures to alleviate free carrier effects such as applying an external DC field [28].

In Figure 7 shows the results obtained for the parameters assumed above. The average error, in this case is $e_{5}=0.31 \mathrm{~dB}$ and $e_{10}=3.83 \mathrm{~dB}$ under the loss independence approximation of Section 4.A, while ignoring TPA-induced loss discussed in Section 4.B yields an average error of the $e_{5}=0.49 \mathrm{~dB}$ and $e_{10}=4.67 \mathrm{~dB}$. The results of Figure 7, indicate that the analytical approximations of the conversion efficiency are still valid even in an hypothetical case where none of the loss mechanisms can be considered dominant. Since the TPA loss cannot be neglected in this case, the aforementioned loss independence approximation outlined in section 4.A, does provide more accurate results.

\section{E. Free-carrier effects in the pulsed regime}

In this section, we briefly examine how the nonlinear loss due to FC generation and its impact on the efficiency $\eta$ can be estimated in the case of a pulsed pump. The time evolution of the FC density, $N_{C}$ is given by [5],

$$
\frac{\partial N_{C}}{\partial t}=\frac{N_{0}-N_{C}}{\tau_{C}}
$$

where $N_{0}=\beta_{\mathrm{TPA}} \tau_{C} S_{p}^{3} P_{p}^{2}(z, t) / 2 \hbar \omega_{p} A_{p p p}{ }^{2}$ is the free carrier density in the continuous wave regime [5,11]. We assume that the input pump signal has a period equal to $T$ and is comprised of rectangular pulses and duration equal to $T_{1}$. As discussed in Section 2 , we can assume that dispersion effects do not significantly affect the pulse shape. Therefore the pump pulse will approximately retain its rectangular shape along the propagation length and only its peak power will decrease because of loss. Solving equation (38) for the $n^{\text {th }}$ pulse period $\left[t_{n}, t_{n+1}\right]$ where $t_{n}=n T$, one finds that,

$$
N_{C}(z, t)=N_{C}\left(z, t_{n}\right) e^{-\left(t-t_{n}\right) / \tau_{C}}+N_{0}\left\{e^{-t_{n} / \tau_{C}}-e^{-\left(t-t_{n}\right) / \tau_{C}}\right\}
$$

during the "on" period of the pulse $t_{n}<t \leq t_{n}+T_{1}$ and

$$
N_{C}(z, t)=N_{C}\left(z, t_{n}+T_{1}\right) e^{-\left(t-t_{n}-T_{1}\right) / \tau_{C}}
$$

if $t_{n}+T_{1}<t \leq t_{n+1}$. In the initial pulse periods (small $n$ ) there will be a gradual build-up of free carriers until one reaches a point where the free carrier density $N_{C}\left(z, t_{n}\right)$ at the start of each period will be the same regardless of $n$. Under this condition one obtains $N_{C}\left(z, t_{n+1}\right)=N_{C}\left(z, t_{n}\right)$ and combining Eqs. (39)-(40) we find that,

$$
N_{c}\left(z, t_{n}\right)=N_{0}\left\{e^{-t_{n} / \tau_{c}}-e^{-T_{1} / \tau_{c}}\right\} \frac{e^{-\left(T-T_{1}\right) / \tau_{c}}}{1-e^{-T / \tau_{c}}}
$$

Using Eqs. (39)-(41), we can easily calculate the average carrier density $N_{\text {avg }}$ inside the pulse duration and use this carrier density 
in the estimations of the loss coefficient in Eq. (9). In Figure 8, the FWM conversion efficiency is shown assuming $T_{1}=50 \mathrm{ps}$ for various pulse repetition rates $1 / T$. In our calculations, we have assumed that the peak pump power is $P_{p}=1 \mathrm{~W}, L=200 \mu \mathrm{m}, \tau_{C}=600 \mathrm{ps}$, $\lambda_{s}=1553.6 \mathrm{~nm}$ and $\lambda_{i}=1549.3 \mathrm{~nm}$. As shown in Figure 8, as the repetition rate becomes smaller, at some point $T$ becomes much larger than $\tau_{\mathrm{C}}$ and the generated free-carriers have the necessary time to fully recombine before the next pulse arrives. Therefore in this regime, $N_{c}\left(z, t_{n}\right) \cong 0$ and the nonlinear losses are due solely to the carriers generated inside the current pulse period which do not depend on $T$ and the repetition rate. As a consequence the efficiency tends to remain constant at small repetition rates. For repetition rates above $1 \mathrm{GHz}$, an exponential degradation of $\eta$ is observed. In this case, carriers generated in the previous pulse duration do not recombine fully and there is a build-up of carriers which increase the nonlinear loss. The figure also shows the efficiency calculated by Eq. (22) when the FC-induced loss is neglected all together. We see that when free-carrier effects are included, the repetition rate has a strong influence in the achieved efficiency and the inclusion of free-carriers induces a penalty ranging from $-8 \mathrm{~dB}$ to $-20 \mathrm{~dB}$.

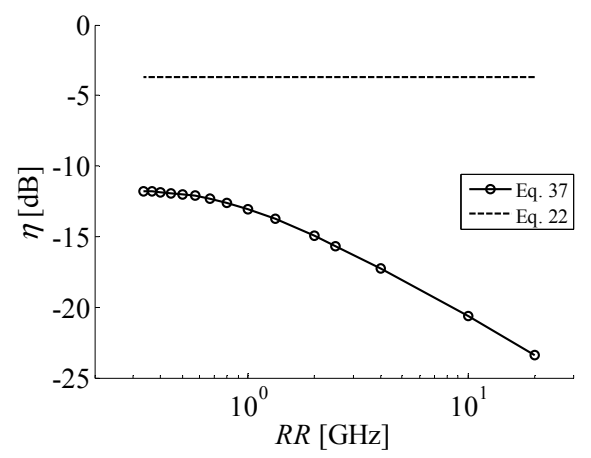

Figure 8. The FWM conversion efficiency of a rectangular pulse train with $T_{1}=50 \mathrm{ps}$ with respect to the pulse repetition rate by applying the Eq. 37 and Eq. 22.

\section{F. Practical implications}

Observing Figures 4 and 6, we deduce that there is no gain on choosing a waveguide length larger than $200 \mu \mathrm{m}$. It is also interesting to note that as shown in Figure 4 when only the TPAinduced loss is incorporated, one can increase the efficiency by increasing the pump power (there are some brighter spots at higher pump power in the surface plots). A different behavior is observed in Figure 6, where the FC effects are dominating, the maximum conversion efficiency is lower and obtained at significant lower pump powers. If the waveguide parameters are such that none of the three loss factors can be neglected then as shown in Figure 7 the efficiency is nearly optimal for a wider range of pump power values. Such considerations are useful when designing a PCW for nonlinear applications and can also be drawn from the semi-analytical results as shown in the figures. In order to gain a better perspective of the impact of the TPA and FCA nonlinear phenomena on the conversion efficiency, we have calculated $\eta_{\max }$ for $\mathrm{PhC}$ waveguides possessing flat variation of the group index around different values previously proposed in the literature. In Table $1, \eta_{\max }$ is calculated for a wavelength detuning of $0.1 \mathrm{~nm}$. The table compares the efficiency values with and without the free-carrier induced absorption assuming a CW regime. The results in Table 1 , show that in larger group index designs, the maximum efficiencies are obtained for shorter lengths since optical losses are increased. In all cases, FCA poses a significant penalty in the achieved efficiencies which is about $9 \mathrm{~dB}$. At larger detunings we have verified that similar penalties due to FC generation are obtained as shown in Table 2 . Note that in Table 2, the design of [33] is not mentioned since the corresponding flat-band region is narrow $(\cong 0.6 \mathrm{~nm})$.

Table 1. Impact of TPA and FCA on the FWM at various $\boldsymbol{n}_{g}$

\begin{tabular}{ccccc}
\hline $\begin{array}{c}\text { Design } \\
\left(n_{g}\right)\end{array}$ & $\begin{array}{c}\eta_{\max }(\mathrm{dB}) \\
\text { using }(22)\end{array}$ & $\begin{array}{c}\eta_{\max }(\mathrm{dB}) \\
\text { using }(37)\end{array}$ & $\mathrm{P}_{\mathrm{p}}(\mathrm{W})$ & $\mathrm{L}(\mu \mathrm{m})$ \\
\hline$[33](100)$ & -17.6 & -25.6 & 0.2 & 25 \\
\hline$[9](60)$ & -13.2 & -23.7 & 0.2 & 75 \\
\hline$[11](30)$ & -11 & -18.5 & 0.4 & 400 \\
\hline
\end{tabular}

Table 2. Impact of detuning on the FWM penalty (with and without FC effects) at various $\boldsymbol{n}_{g}$

\begin{tabular}{ccc}
\hline$\Delta \Lambda(\mathrm{nm})$ & $\begin{array}{c}\text { FC-penalty }(\mathrm{dB}) \\
n_{g}=30[9]\end{array}$ & $\begin{array}{c}\text { FC-penalty }(\mathrm{dB}) \\
n_{g}=60[11]\end{array}$ \\
\hline 1 & -8.4 & -14.8 \\
\hline 2 & -8.5 & -16.2 \\
\hline 3 & -7.6 & -14.1 \\
\hline
\end{tabular}

\section{CONCLUSIONS}

In this paper, we have provided approximate expressions of the degenerate FWM conversion efficiency in photonic crystal waveguides. In order to investigate the validity of the derived expressions we have compared their values against numerical solution of the coupled propagation equations for a wide set of parameter values. Our approach is based on modifying the FWM efficiency obtained in the lossless problem in order to account for the pump variation across the waveguide and the losses experienced by the idler wave. This approximation allows us to obtain simple analytical expressions of the FWM conversion efficiency. The model incorporates the effect of linear losses and the loss induced by TPA and free carrier effects. In all cases we have shown that the analytical expressions accurately predict the efficiency values $-5 \mathrm{~dB}$ away from its maximum value. As we move to lower efficiency values, the error increases but the formulas can still be used with reasonable accuracy. These expressions for the FWM efficiency are expected to be very useful in the design of photonic crystal waveguides considered for nonlinear signal applications.

\section{ACKNOWLEDGEMENTS}

This research has been co-financed by the European Union (European Social Fund - ESF) and Greek national funds through the Operational Program "Education and Lifelong Learning" of the National Strategic Reference Framework (NSRF) - Research Funding Program: Heracleitus II. Investing in knowledge society through the European Social Fund.

\section{REFERENCES}

1. Y. Gong, J. Huang, K. Li, N. Copner, J. J. Martinez, L. Wang, T. Duan, W. Zhang, and W. H. Loh, "Spoof four wave mixing for all-optical wavelength conversion," Opt. Express 20, 24030-24037 (2012).

2. R. Slavik, F. Parmigiani, J. Kakande, C. Lundstrom, M. Sjodin, P. A. Andrekson, R. Weerasuriya, S. Sygletos, A. D. Ellis, L. Gruner-Nielsen, D. Jakobsen, S. Herstrom, R. Phelan, J. O'Gorman, A. Bogris, D. Syvridis, S. Dasgupta, P. Petropoulos, and D. J. Richardson, "All-optical phase 
and amplitude regenerator for next-generation telecommunications systems," Nat. Photonics 4, 690-695 (2010).

3. Q. Lin, O. J. Painter, and G. P. Agrawal, "Nonlinear optical phenomena in silicon waveguides: Modeling and applications," Opt. Express 15, 16604-16644 (2007).

4. R. Salem, M. A. Foster, A. C. Turner, D. F. Geragthy, M. Lipson, and A. L.Gaeta, "Signal regeneration using lowpower four-wave mixing on silicon chip," Nat. Photonics 2(1), 465-473 (2008).

5. S. Rawal, R. K. Sinha, R. M. De La Rue, "Silicon-on" insulator photonic miniature devices with slow light enhanced third-order nonlinearities," J. Nanophoton. 6(1), 063504, doi:10.1117/1.JNP.6.063504 (2012).

6. B. Corcoran, M. D. Pelusi, C. Monat, J. Li, L. O'Faolain, T. F. Krauss, and B. J. Eggleton, "Ultracompact 160 Gbaud all-optical demultiplexing exploiting slow light in a engineered silicon photonic crystal waveguide" Opt. Letters 36, 1728-1730 (2011).

7. F. Morichetti, A. Canciamilla, C. Ferrari, A. Samarelli, M. Sorel, and A. Melloni, "Travelling-wave resonant four-wave mixing breaks the limits of cavity-enhanced all-optical wavelength conversion," Nat. Communications 2, 296 doi: 10.1038/ncomms1294, (2011).

8. J. Li, L. O'Faolain, and T. F. Krauss, "Four-wave mixing in slow light photonic crystal waveguides with very high group index", Opt. Express 20, 17474-17479 (2012).

9. J. Li, L. O’Faolain, S. A. Schulz, T. F. Krauss, "Low loss propagation in slow light photonic crystal waveguides at group indices up to 60", Photonics and Nanostructures Fundamentals and Applications 10, 589-593 (2012).

10. G. P. Agrawal, Non-Linear Fiber Optics, $4^{\text {th }}$ edition (Academic 2007).

11. T. Chen, J. Sun, and L. Li, "Modal theory of slow light enhanced third-order nonlinear effects in photonic crystal waveguides," Opt. Express 20, 20043-20058 (2012).

12. S. A. Schulz, L. O'Faolain, D. M. Beggs, T. P. White, A. Melloni, and T. F. Krauss, "Dispersion engineered slow light in photonic crystals: a comparison," J. Optics 12, 104004, doi:10.1088/2040-8978/12/10/104004 (2010).

13. S. Roy, M. Santagiustina, P. Colman, S. Combrie, A. De Rossi, "Modeling the dispersion of the nonlinearity in slow mode photonic crystal waveguides," IEEE Photonics Journal 4, 224-233 (2012).

14. P. Kanakis, T. Kamalakis, and T. Sphicopoulos, "Optimization of the storage capacity of slow light photonic crystal waveguides," Opt. Letters 37, 4585-4587 (2012).

15. L. O'Faolain, S. A. Schulz, D. M. Beggs, T. P. White, M. Spasenovic, L. Kuipers, F. Morichetti, A. Melloni, S. Mazoyer, J. P. Hugonin, P. Lalanne, and T. F. Krauss, "Loss engineered slow light waveguides," Opt Express 18, 27627-27638 (2010).

16. P. Kanakis, T. Kamalakis, and T. Sphicopoulos, "Numerical analysis of soliton propagation in photonic crystal slab waveguides for signal processing application," JOSA B 29, 2787-2796, (2012).

17. L. Yin, and G. Agrawal, "Impact of two photon absorption on self-phase modulation in silicon waveguides," Opt. Letters 32, 2031-2033 (2007).

18. C. Monat, B. Corcoran, M. Ebnali-Heidari, C. Grillet, B. J. Eggleton, T. P.White, L. O'Faolain, and T. F. Krauss, "Slow light enhancement of nonlinear effects in silicon engineered photonic crystal waveguides," Opt. Express 17, 2994-2953 (2009).

19. B. Corcoran, C. Monat, D. Pudo, B. J. Eggleton, T. F. Krauss, D. J. Moss, L. O'Faolain, M. Pelusi and T. P. White, "Nonlinear loss dynamics in a silicon slow-light photonic crystal waveguide," Opt. Letters 35, 1073-1075 (2010).

20. J. F. McMillan, M. Yu, D. Kwong, and C. Wong, "Observation of four-wave mixing in slow light silicon photonic crystal wavguides," Opt. Express 18, 15484-15497 (2010).

21. C. Monat, M. Ebnali-Heidari, C. Grillet, B. Corcoran, B. J. Eggleton, T. P. White, L. O'Faolain, J. Li, and T. F. Krauss, "Four-wave mixing in slow light engineered silicon photonic crystal waveguides," Opt. Express 18, 2291522915 (2010).

22. M. Santagiustina, C. G. Someda, G. Vadala, S. Combrie, and A. De Rossi, "Theory of slow light enhanced four-wave mixing in photonic crystal waveguides," Opt. Express 18, 21024-21029 (2010).

23. K. Lengle, L. Bramerie, M. Gay, M. Costa e Silva, S. Lobo, J. Simon, P. Colman, S. Combrie, and A. de Rossi, "Investigation of FWM in dispersion-engineered GaInP photonic crystal waveguides", Opt. Express 20, 1615416165, (2012).

24. T. Vallaitis, S. Bogatscher, L. Alloatti, P. Dumon, R. Baets, M. L. Scimeca, I. Biaggio, F. Diederich, C. Koos, W. Freude, and J. Leuthold, "Optical properties of highly nonlinear silicon-organic hybrid (SOH) waveguide geometries," Opt. Express 17, 17357-17368 (2009).

25. K. Suzuki and T. Baba, "Nonlinear light propagation in chalcogenide photonic crystal slow light waveguides," Opt. Express 18, 26675-26685 (2010).

26. S. Roy, A. Willinger, S. Combrie, A. De Rossi, G. Eisenstein and M. Santaguistina, "Narrowband optical parametric gain in slow mode engineered GaInP photonic crystal wavegudies", Opt. Letters 37, 2919-2921 (2012).

27. H. Rong, R. Jones, A. Liu, O. Cohen, D. Hak, A. Fang, and M. Paniccia, "A continuous-wave Raman silicon laser," Nature 433, 725-728 (2005).

28. A. C. Turner-Foster, M. A. Foster, J. S. Levy, C. B. Poitras, R. Salem, A. L. Gaeta, and M. Lipson, "Ultrashort free-carrier lifetime in low-loss silicon nanowaveguides," Opt. Express 18, 3582-3591 (2010).

29. J. R. Dormand, and P. J. Prince, "A family of embedded Runge-Kutta formulae," J. Comp. Appl. Math. 6, 19-26 (1980).

30. K. Inoue, H. Oda, N. Ikeda, and K. Asakawa, "Enhanced third-order nonlinear effects in slow-light photonic-crystal slab waveguides of line defect," Opt. Express 17, 7206-7216 (2009).

31. B. Corcoran, T. D. Vo, M. D. Pelusi, C. Monat, D. Xu, A. Densmore, R. Ma, S. Janz, D. J. Moss, and B. J. Eggleton, "Silicon nanowire based radio-frequency spectrum analyzer," Opt. Express 18, 20190-20200 (2010).

32. I. D. Rukhlenko, M. Premarante, and G. P. Agrawal, "Nonlinear silicon photonics: analytical tools," J. Sel. Top. Quantum Electron. 16, 200-215 (2010).

33. J. Li, T. P. White, L. O'Faolain, A. Gomez-Iglesias, and T. F. Krauss, "Systematic design of flat band slow light in photonic crystal waveguides," Opt. Exp., vol. 16, 6227-6232 (2008). 